\title{
Профилирование компонент подвижности вблизи гетерограниц тонких пленок кремния
}

\author{
(c) Э.Г. Зайцева ", О.В. Наумова, Б.И. Фомин \\ Институт физики полупроводников им. А.В. Ржанова Сибирского отделения Российской академии наук, \\ 630090 Новосибирск, Россия \\ E-mail: zayceva@isp.nsc.ru
}

Поступила в Редакцию 26 сентября 2019 г.

В окончательной редакции 15 октября 2019 г.

Принята к публикации 15 октября 2019 г.

\begin{abstract}
Предложен метод профилирования компонент эффективной подвижности носителей заряда $\mu_{\mathrm{eff}}$ определяемых рассеянием на поверхностных фононах и микрорельефе границ раздела тонкая пленка/диэлектрик. Метод основан на управляемой локализации носителей заряда относительно тестируемой гетерограницы за счет взаимосвязи потенциалов на противоположных сторонах пленки (coupling-эффекта). Предложенный метод позволяет независимо выделять компоненты подвижности вблизи разных гетерограниц пленок. Использование его при исследовании подвижности электронов в пленках кремний-на-изоляторе позволило получить информацию о шероховатости границы раздела и структурном совершенстве ультратонкого (1-3 нм) слоя кремния вблизи скрытой границы раздела пленка/диэлектрик.
\end{abstract}

Ключевые слова: тонкие пленки, кремний-на-изоляторе, подвижность, гетерограница.

DOI: $10.21883 /$ FTP.2020.02.48891.9272

\section{1. Введение}

Для большого класса современных устройств на основе пленок кремний-на-изоляторе (КНИ) (тонкопленочные транзисторы, волноводы и оптические модуляторы, химические и биологические сенсоры и др. [1-6]) свойства внутренней границы раздела (ГР) пленки и скрытого окисла (BOX - buried oxide), как и свойства структуры пленка/диэлектрик на ее поверхности, являются в равной степени значимыми для функционирования приборов. Свойства внутренней ГР Si/BOX зависят от технологии изготовления структур, в частности изготовления пластин КНИ, и могут быть модифицированы при изготовлении приборов [7,8]. Поэтому одной из ключевых задач для широкого класса приборов на основе пленок является определение и контроль параметров внутренней ГР. Эта проблема особенно актуальна для тонкопленочных структур, в которых потенциалы на противоположных ГР пленки взаимосвязаны (наблюдается так называемый coupling-эффект [9-11]). Поэтому разработка методов независимой характеризации свойств ГР тонкопленочных структур имеет особое значение.

Подвижность носителей заряда является фундаментальным параметром материала, который позволяет характеризовать степень совершенства систем пленка/диэлектрик. Однако подвижность носителей заряда $\mu_{\text {eff }}$ является многокомпонентной функцией. В тонких пленках в условиях coupling-эффекта подвижность $\mu_{\text {eff }}$ может быть записана с помощью следующего выражения:

$$
\mu_{\mathrm{eff}}=\left(\frac{1}{\mu_{b}}+\frac{1}{\mu_{s 1}}+\frac{1}{\mu_{s 2}}\right)^{-1},
$$

где $\mu_{b}$ - объемная компонента подвижности, определяемая рассеянием на ионизованной примеси $\left(\mu_{b_{-}}\right)$ и на фононах $\left(\mu_{b \_p h}\right), \mu_{s 1}$ и $\mu_{s 2}-$ компоненты подвижности, связанные с рассеянием носителей на флуктуациях потенциала вблизи каждой из гетерограниц. Компоненты $\mu_{s 1}$ и $\mu_{s 2}$ определяются рассеянием на поверхностных состояниях и на заряде в диэлектрике $\left(\mu_{s_{-} \mathrm{C}}\right)$, рассеянием на поверхностных фононах $\left(\mu_{s_{-} \mathrm{ph}}\right)$ и рассеянием на микрорельефе ГР $\left(\mu_{s r}\right)[12,13]$. Для характеризации системы полупроводник/диэлектрик наиболее интересны компоненты $\mu_{s \_ \text {ph }}$ и $\mu_{s r}$, которые определяются структурным совершенством пленок вблизи гетерограниц. Выделить компоненты $\mu_{s_{-} \text {рh }}$ и $\mu_{s r}$ на основе уравнения (1) невозможно. Однако выражение (1) может быть упрощено путем минимизации вклада компонентов подвижности.

Известно, что вклад механизмов рассеяния в значение $\mu_{\text {eff }}$ определяется распределением концентрации носителей заряда по толщине $\mathrm{Si}$ относительно гетерограниц, $N\left(t_{\mathrm{Si}}\right)$; при использовании тонкопленочных МОП транзисторов (металл-окисел-полупроводник) в условия coupling-эффекта распределение $N\left(t_{\mathrm{Si}}\right)$ определяется напряжением на двух затворах (BG и TG, см. вставку к рис. 1) [14,15]. Варьирование напряжений на затворах приводит к значительному перераспределению носителей заряда как вблизи гетерограниц, так и в объеме пленки (рис. 1). Так, для $n$-канальных КНИ транзисторов с индуцированным положительным напряжением на затворе BG электронным каналом проводимости можно отрицательным напряжением на противоположном затворе TG практически полностью сосредоточить электроны вблизи ГР пленка/ВОХ (рис. 1, кривая 1). В работе [16] было показано, что при условиях сильной инверсии-обогащения со стороны противоположных гетерограниц пленки и низкой концентрации легирующей примеси компонентами $\mu_{s 2}, \mu_{\mathrm{C}}$ и $\mu_{s} 1_{-} \mathrm{c}$ в $\mu_{\text {eff }}$ можно 


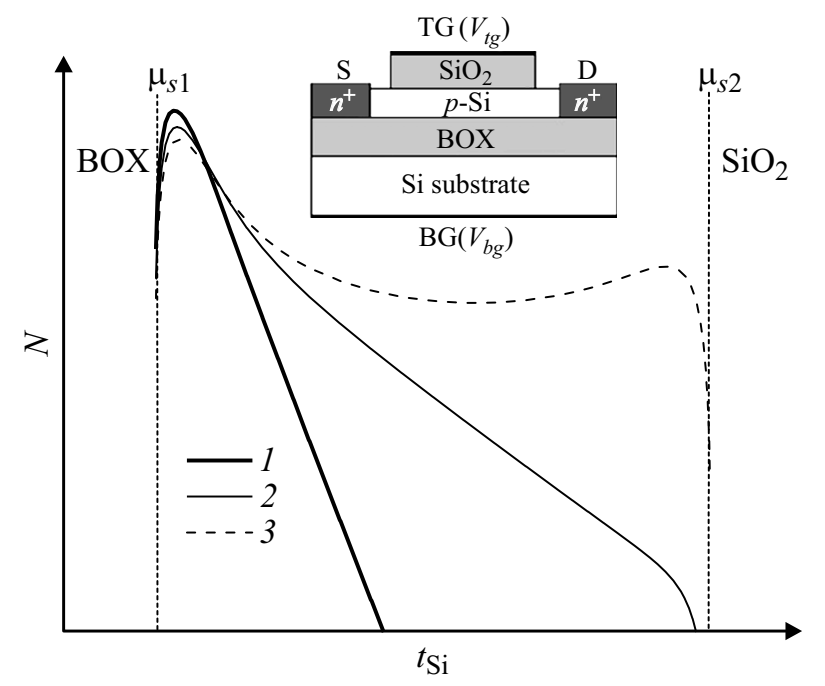

Рис. 1. Схематическое представление распределения носителей заряда $N\left(t_{\mathrm{Si}}\right)$ в КНИ МОП транзисторах при режимах обогащения (1), обеднения (2) и инверсии пленки со стороны затвора TG (3). S - исток, D - сток. На вставке - схематическое изображение тонкопленочного КНИ МОП транзистора.

пренебречь. Тогда выражение (1) запишется в виде

$$
\begin{aligned}
\mu_{\mathrm{eff}} & \approx\left(\frac{1}{\mu_{b}}+\frac{1}{\mu_{s 1}}\right)^{-1} \\
& =\left(\frac{1}{\mu_{b_{-} \mathrm{ph}}}+\frac{1}{\mu_{s 1 \_\mathrm{ph}}}+\frac{1}{\mu_{s r 1}}\right)^{-1} .
\end{aligned}
$$

Понятно, что выражение (2) существенно упрощает выделение компонент подвижности $\mu_{s_{-} \text {рh }}$ и $\mu_{s r}$. Таким образом, coupling-эффект может быть использован для перераспределения носителей по пленке, минимизации компонент $\mu_{\text {eff }}$ и для профилирования компонент $\mu_{s \_ \text {ph }}$ и $\mu_{s r}$.

Цель данной работы заключалась в разработке метода независимой характеризации компонент подвижности $\mu_{s \_ \text {ph }}$ и $\mu_{s r}$ вблизи гетерограниц тонких пленок. Для этого coupling-эффект был использован для выделения и получения зависимостей $\mu_{s \_p h}$ и $\mu_{s r}$ от центроида индуцированных носителей заряда. Данный метод был применен для определения компонент подвижности вблизи внутренней ГР Si/BOX тонких пленок КНИ.

\section{2. Экспериментальные данные и методика}

В работе использовались пленки $p$-типа проводимости с концентрацией акцепторов в отсеченном слое кремния $N_{a}=2 \cdot 10^{16} \mathrm{~cm}^{-3}$, полученные от двух производителей: SOI-1 и SOI-2. Толщина пленок составляла $t_{\mathrm{Si}}=30$ нм, толщина ВОХ $t_{\mathrm{BOX}}=200$ нм. На их основе были изготовлены $n$-канальные КНИ МОП транзисторы с длиной канала $L=10$ мкм, шириной $W=3$ мкм. Толщина подзатворного окисла транзисторов составляла $t_{\text {ох }}=200 \mathrm{Hм}$.

Эффективная подвижность носителей (электронов) $\mu_{\text {eff }}$ определялась из сток-затворных характеристик транзисторов $I_{d s}\left(V_{b g}\right)$. Электронный канал проводимости индуцировался вблизи внутренней ГР $\mathrm{Si} / \mathrm{BOX}$ напряжением на затворе $\mathrm{BG}$, роль которого играла подложка структур КНИ, т.е. BG-затвор использовался в экспериментах в качестве управляющего. Напряжение на дополнительном затворе $\mathrm{TG}, V_{t g}$, использовалось в качестве параметра. Измерения проводились в диапазоне температур $77-300 \mathrm{~K}$. Значения подвижности $\mu_{\text {eff }}$ и плотность индуцированных носителей заряда в канале $N_{e}$ рассчитывались из зависимостей $I_{d s}\left(V_{b g}\right)$ при использовании стандартных выражений $[17,18]$

$$
\begin{gathered}
\mu_{\mathrm{eff}}=\frac{I_{d s}}{(W / L) C_{\mathrm{BOX}} V_{d s}\left(V_{b g}-V_{\mathrm{th}}\right)}, \\
N_{e}=\frac{C_{\mathrm{BOX}}}{q}\left(V_{b g}-V_{\mathrm{th}}\right),
\end{gathered}
$$

где $I_{d s}$ - ток сток-исток, $V_{d s}-$ напряжение на стоке, $V_{\text {th }}-$ пороговое напряжение транзистора, $C_{\text {вох }}-$ емкость скрытого окисла, $q$ - заряд электрона.

Компоненты $\mu_{s \_p h}$ и $\mu_{s r}$ определялись из температурных зависимостей $\mu_{\text {eff }}(T)$ при значениях $N_{e} \geq 2 \cdot 10^{12} \mathrm{~cm}^{-2}$. Условие большой плотности носителей заряда в индуцированном канале проводимости использовалось, чтобы пренебречь кулоновской составляющей $\mu_{s_{-} \text {С }}$ в $\mu_{\text {eff, }}$ согласно работе [12]. Для выделения компонент $\mu_{s \_ \text {ph }}$ и $\mu_{s r}$ из температурных зависимостей $\mu_{\mathrm{eff}}$ использовалась модель Ломбарди [19], согласно которой для систем $\mathrm{Si} / \mathrm{SiO}_{2} \mu_{b_{-} \mathrm{ph}}(T)=$ $=1417 \cdot(T / 300)^{-2.5}, \mu_{s r}=$ const, $\mu_{s \_p h}(T)=a+b T^{-1}$. Здесь $a$ и $b-$ подгоночные коэффициенты $\left(a \propto E_{\text {eff }}^{-1}\right.$ и $b \propto E_{\text {eff }}^{-1 / 3}$ ). Отметим, что в области сильных полей (или при больших значениях $\left.N_{e}[20]\right), \mu_{s_{-} \mathrm{ph}}(T)$ может быть аппроксимирована зависимостью $\mu_{s \_p h}(T)=b T^{-1}$.

Численное моделирование в программе SDevice из пакета TCAD Sentaurus было использовано для аппроксимации экспериментальных зависимостей $I_{d s}\left(V_{b g}\right)$ при разных значениях $V_{t g}$ и расчета распределения свободных носителей заряда по толщине пленки $N\left(t_{\mathrm{Si}}\right)$. Центроид заряда свободных носителей $\rho$ определялся из их распределения $N\left(t_{\mathrm{Si}}\right)$, как в [21]:

$$
\rho=\int_{0}^{\infty} t_{\mathrm{Si}} N\left(t_{\mathrm{Si}}\right) d t_{\mathrm{Si}} / \int_{0}^{\infty} N\left(t_{\mathrm{Si}}\right) d t_{\mathrm{Si}} .
$$

Центроид заряда $\rho$ определялся для диапазона значений $N_{e}=(2-8) \cdot 10^{12} \mathrm{~cm}^{-2}$. Для сравнения аналогичные зависимости $\mu_{s r}(\rho)$ и $\mu_{s_{-} \mathrm{ph}}(\rho)$ были определены для классической ГР $\mathrm{Si} / \mathrm{SiO}_{2}$, сформированной термическим окислением объемного кремния. Для этого использовались экспериментальные зависимости $\mu_{\mathrm{eff}}\left(E_{\mathrm{eff}}\right)$, полученные в диапазоне температур 77-447 K для МОП транзисторов на объемном кремнии в работе [12]. Распределение носителей $N\left(t_{\mathrm{Si}}\right)$ при соответствующих значениях 
$E_{\text {eff }}$ также рассчитывалось с использованием программы TCAD. Отметим, что полученные в работе [12] зависимости $\mu_{\text {eff }}\left(E_{\text {eff }}\right)$ считаются эталонными, полученными для высококачественной системы $\mathrm{Si} / \mathrm{SiO}_{2}$, и используются во многих работах для сравнения.

\section{3. Результаты и обсуждение}

На рис. 2 показаны температурные зависимости $\mu_{\text {eff }}$ для электронов вблизи ГР $\mathrm{Si} / \mathrm{BOX}$ пленок SOI-1 и SOI-2, полученные при фиксированном значении $N_{e}=6 \cdot 10^{12} \mathrm{~cm}^{-2}$ в канале и при условии обогащения пленки со стороны дополнительного затвора TG. На рис. 2 также показаны аппроксимации температурных зависимостей $\mu_{\text {eff }}$ и компоненты $\mu_{s} 1 \_$p и $\mu_{s r 1}$, полученные при использовании выражения (2). Для сравнения на рис. 2 показаны аналогичные зависимости, полученные для классической $Г \mathrm{P} \mathrm{Si} / \mathrm{SiO}_{2}$ на основе данных работы [12].

Из рис. 2 видно, что: 1) для пленок КНИ разница в значениях компонент $\mu_{s \_ \text {ph }}$ и $\mu_{s r}$ существенно больше, чем в значениях $\left.\mu_{\mathrm{eff}} ; 2\right)$ в пленках SOI-1 подвижность, связанная с рассеянием на фононах вблизи внутренней ГР меньше, чем в пленках SOI-2, т.е. $\mu_{s 1 \_p h}(\mathrm{SOI}-1)<\mu_{s 1 \_p h}(\mathrm{SOI}-2)$; для компоненты, связанной с рассеянием на микрорельефе ГР $\mathrm{Si} / \mathrm{BOX}$ имеет место обратное соотношение $\mu_{s r 1}(\mathrm{SOI}-1)>\mu_{s r}$ (SOI-2); 3) значения $\mu_{\text {eff }}, \mu_{s \_p h}$ и $\mu_{s r}$ вблизи скрытой ГР пленок КНИ меньше, чем соответствующие значения для классической ГР. Такое поведение подвижности может быть связано как с разным структурным качеством систем $\mathrm{Si} / \mathrm{SiO}_{2}$, так и с разной локализацией электронов относительно гетерограниц.

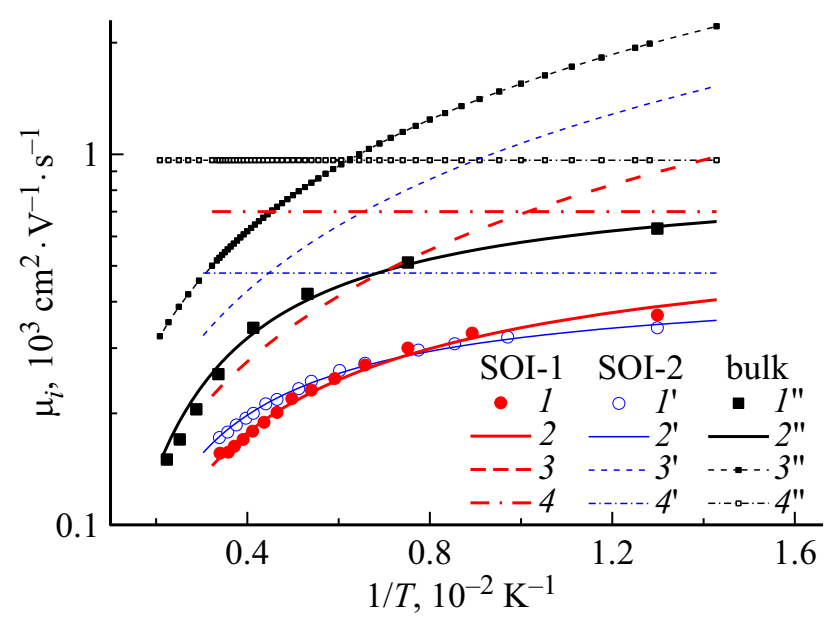

Рис. 2. Зависимости $\mu_{\mathrm{eff}}\left(T^{-1}\right) \quad\left(1,1^{\prime}, 1^{\prime \prime}\right)$, их аппроксимации $\left(2,2^{\prime}, 2^{\prime \prime}\right), \quad$ компоненты $\mu_{s_{-} \text {ph }}\left(T^{-1}\right) \quad\left(3,3^{\prime}, 3^{\prime \prime}\right)$ и $\mu_{s r}\left(T^{-1}\right)\left(4,4^{\prime}, 4^{\prime \prime}\right)$ для ГР $\mathrm{Si} / \mathrm{BOX}$ в пленках $\mathrm{SOI}-1(1-4)$, SOI-2 $\left(1^{\prime}-4^{\prime}\right)$ при $N_{e}=6 \cdot 10^{12} \mathrm{~cm}^{-2}$ и режиме обогащения пленки со стороны ТG $\left(V_{t g}=-40 \mathrm{~B}\right)$, для классической ГР 〈объемный $\mathrm{Si}\rangle / \mathrm{SiO}_{2}\left(1^{\prime \prime}-4^{\prime \prime}\right)$; кривая $1^{\prime \prime}$ получена по данным работы [12], $E_{\text {eff }}=106 \mathrm{~B} / \mathrm{cm}$.

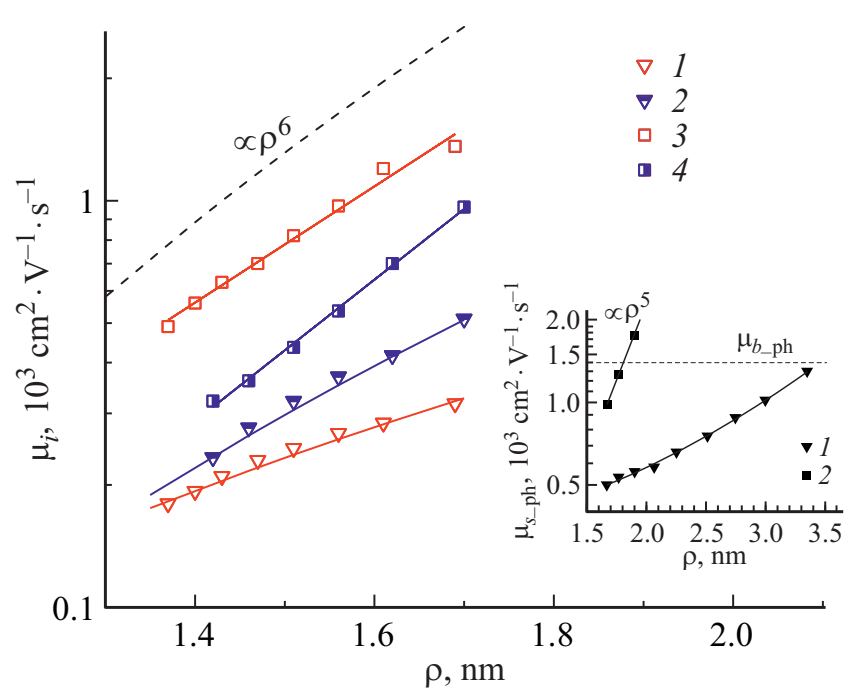

Рис. 3. Зависимости компонент подвижности $\mu_{s \_ \text {ph }}(1,2)$ и $\mu_{s r}(\rho)(3,4)$ вблизи ГР $\mathrm{Si} / \mathrm{BOX}$ в пленках $\mathrm{SOI}-1$ и SOI-2 coответственно. На вставке - зависимости $\mu_{s \_ \text {ph }}(\rho)(1)$ и $\mu_{s r}(\rho)(2)$ вблизи классической ГР $\langle$ объемный $\mathrm{Si}\rangle / \mathrm{SiO}_{2}$. Пунктирной линией показано значение $\mu_{b_{-} \text {ph. }} T=300 \mathrm{~K}$.

На рис. 3 показаны зависимости компонент $\mu_{s \_p h}$ и $\mu_{s r}$ от центроида заряда $\rho$ для пленок SOI-1, SOI-2 и для классической границы раздела 〈объемный $\mathrm{Si}\rangle / \mathrm{SiO}_{2}$ (вставка). Видно, что значения подвижности для пленок КНИ и для системы 〈объемный $\mathrm{Si}\rangle / \mathrm{SiO}_{2}$ соответствуют разным диапазонам $\rho$. Минимальное достижимое значение $\rho$ вблизи классической ГР составляет $\sim 1.7 \mathrm{HM}$ (ограничено пробоем подзатворного диэлектрика), в то время как для пленок КНИ при условии экранирования дополнительной ГР (режим обогащения пленки со стороны TG) значение $\rho$ было определено в диапазоне $1.3-1.7 \mathrm{HM}$.

Для классической системы 〈объемный $\mathrm{Si}\rangle / \mathrm{SiO}_{2}$ зависимости $\mu_{s \_ \text {ph }}(\rho)$ монотонно возрастают с увеличением $\rho$ и имеют экспоненциальный характер, $\mu_{s \_p h} \propto \exp (\rho)$. Такая зависимость может быть объяснена экспоненциальной зависимостью деформационного потенциала, определяемого скоростью рассеяния электронов на акустических фононах, от расстояния до границы раздела $\mathrm{Si} / \mathrm{SiO}_{2}$ [22]. Из вставки к рис 3 видно, что для системы $\langle$ объемный $\mathrm{Si}\rangle / \mathrm{SiO}_{2}$ при $\rho \approx 3.5$ нм компонента

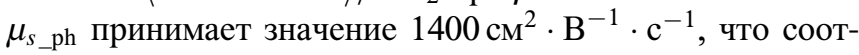
ветствует максимальному значению подвижности, определяемой рассеянием на фононах в объеме материала, $\mu_{b \_p h}$, при $T=300 \mathrm{~K}$. Для системы $\mathrm{Si} / \mathrm{BOX}$ исследуемых пленок характер зависимостей $\mu_{s \_p h}(\rho)$ не изменяется значение подвижности уменьшается с уменьшением $\rho$, что качественно объясняется увеличением деформационного потенциала (структурных нарушений) по направлению к границе раздела. Зависимости $\mu_{s \_p h}(\rho)$ могут быть аппроксимированы степенной функцией $\mu_{s \_ \text {ph }} \propto \rho^{n}$, где показатель $n$ имеет значения 2.7 и 4.3 для пленок SOI-1 и SOI-2 соответственно. 
Очевидно, что с уменьшением $\rho$ увеличивается роль флуктуаций потенциала, вызванных шероховатостью гетерограницы, в рассеяние носителей. Поэтому значения $\mu_{s r}$ так же уменьшаются с уменьшением расстояния до ГР. Зависимости $\mu_{s r}(\rho)$, как видно из рис. 3 , описываются степенной функцией $\mu_{s r} \propto \rho^{m}$ с показателем $m=5$ для классической ГР 〈объемный $\mathrm{Si}\rangle / \mathrm{SiO}_{2}$ и $m=5.5-6$ для гетерограниц в тонких пленках КНИ. Отметим, что показатель $m=6$ характерен для рассеяния носителей на шероховатости гетерограниц в ультратонких пленках КНИ [23].

Из рис. 3 видно, что для исследуемых пленок КНИ соотношения $\mu_{s 1 \_p h}(\mathrm{SOI}-1)<\mu_{s 1 \_p h}(\mathrm{SOI}-2)$ и $\mu_{s r 1}(\mathrm{SOI}-1)>\mu_{s r 1}(\mathrm{SOI}-2)$ сохраняются во всем диапазоне $\rho$. Это позволяет заключить, что структурное качество пленок SOI-2, определяющее рассеяние на фононах вблизи BOX, лучше, чем в пленках SOI-1. Однако шероховатость $(\Delta)$ ГР Si/BOX в SOI-2 больше, чем в SOI-1. Полученные результаты были подтверждены результатами исследования методом высокоразрешающей электронной микроскопии (ВРЭМ), согласно которым шероховатость ГР $\mathrm{Si} / \mathrm{BOX}$ в пленках $\mathrm{SOI}-1$ составляла $\Delta \approx 1 \mathrm{Hм}$, а в пленках SOI-2 $\Delta \approx 2.4 \mathrm{нм}$.

Таким образом, предложенный подход позволяет вы-

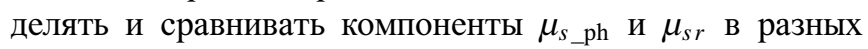
пленках, выявляя доминирующий механизм рассеяния. Однако такое сравнение $\mu_{s \_p h}$ и $\mu_{s r}$ в пленках правомерно проводить при условиях равных значений $\rho$, при одинаковом распределении носителей заряда. В данной работе были рассмотрены КНИ МОП транзисторы с одинаковыми конструктивными параметрами, именно с одинаковыми толщинами пленки кремния и окружающих ее диэлектриков. В таких транзисторах одно и то же напряжение на TG и BG создает одинаковое распределение носителей относительно гетерограниц, соответственно одинаковое значение $\rho$. Поэтому использование зависимостей $\mu_{\mathrm{eff}}(\rho)$ для сравнения свойств гетерограниц правомерно. Однако в транзисторах с разными конструктивными параметрами распределение носителей заряда может различаться даже при одном и том же значении $\rho$. Поэтому возникает вопрос, сохраняется ли соответствие между распределением $N\left(t_{\mathrm{Si}}\right)$ и центроидом заряда $\rho$ в структурах с разными конструктивными параметрами, - например, с разной толщиной пленок кремния.

На рис. 4 представлено распределение электронов $N\left(t_{\mathrm{Si}}\right)$ в тонкопленочных МОП транзисторах с толщиной пленок КНИ $t_{\mathrm{Si}}=20-50$ нм при режимах обогащения и обеднения пленки со стороны TG. Для сравнения аналогичные зависимости представлены для КНИ транзисторов с толщиной 50 нм с поверхностными состояниями плотностью $D_{i t}=10^{12} \mathrm{~cm}^{-2}$ на ГР $\mathrm{Si} / \mathrm{BOX}$. Из рис. 4 видно, что при варьировании толщины пленок КНИ распределение $N\left(t_{\mathrm{Si}}\right)$ сохраняется при условии обеднения пленок со стороны TG. При этом наличие ловушек на ГР $\mathrm{Si} / \mathrm{BOX}$ не оказывает влияния на распределение электронов в пленке КНИ, как при режиме

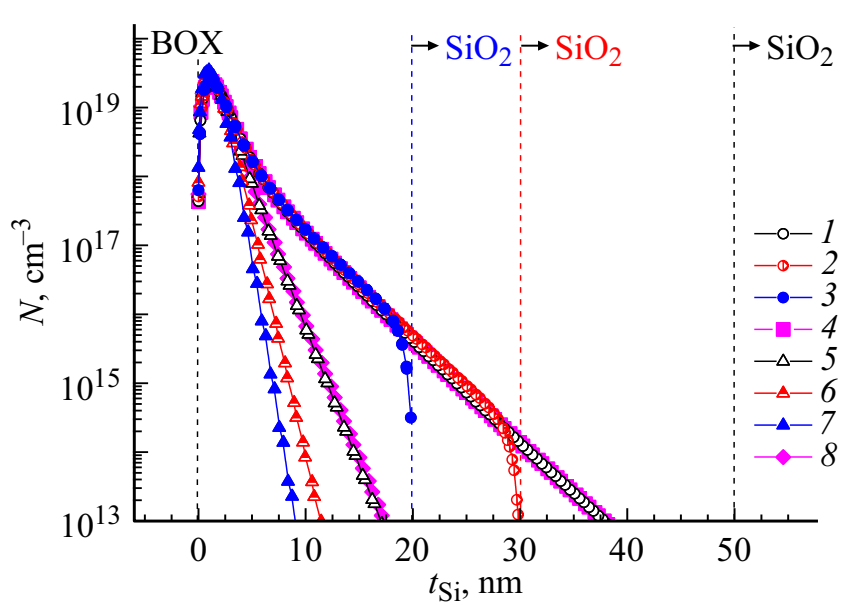

Рис. 4. Распределение электронов $N\left(t_{\mathrm{Si}}\right)$ в КНИ МОП транзисторах при режимах обеднения $(1-4)$ и обогащения пленки со стороны TG $(5-7)$ при $t_{\mathrm{Si}}$, нм: $(1,5)-50,(2,6)-30,(3,7)-$ $20,(4,8)-50$ (на ГР Si/BOX присутствуют поверхностные состояния, плотность $\left.D_{i t}=10^{12} \mathrm{~cm}^{-2}\right) . T=300 \mathrm{~K}$.

обеднения, так и при режиме обогащения пленки со стороны TG. Таким образом, в тонких пленках разной толщины сопоставление подвижности правомерно при условии обеднения пленки со стороны дополнительного затвора (в данном случае затвора TG).

Отметим, что основным условием минимизации вклада компонент подвижности в выражении (1) является условие экранирования противоположной ГР. Это условие выполняется в режиме сильной инверсии/обогащения с противоположных сторон пленки. Однако в ультратонких пленках, для которых характерен super-coupling-эффект, этот режим недостижим [24]. Таким образом, ограничением данного метода при использовании режима сильной инверсии/обогащения является super-coupling-эффект. При сравнении компонент подвижности в режиме сильной инверсии/обеднения тонких пленок необходимым условием является низкая плотность состояний на гетерогранице, противоположной тестируемой.

\section{4. Заключение}

В работе представлен метод профилирования компонент подвижности, связанных с рассеянием на фононах вблизи поверхности, $\mu_{s \_ \text {ph }}$ и на микрорельефе гетерограниц, $\mu_{s r}$. Определены условия применимости метода - режим „сильная инверсия-обогащение“ со стороны противоположных гетерограниц или режим „сильная инверсия-обеднение“ пленок при условии низкой плотности поверхностных состояний со стороны ГР, противоположной тестируемой.

Представленный метод был апробирован для характеризации внутренней ГР $\mathrm{Si} / \mathrm{BOX}$ тонкопленочных КНИ МОП транзисторов. Анализ зависимостей $\mu_{s \_p h}(\rho)$ и $\mu_{s r}(\rho)$ показал, что структурные свойства пленок вбли- 
зи ГР $\mathrm{Si} / \mathrm{BOX}$ могут значительно различаться, несмотря на близкие значения эффективной подвижности.

Показано, что зависимости $\mu_{s \_p h}(\rho)$ и $\mu_{s r}(\rho)$ в пленках КНИ можно аппроксимировать степенными функциями $\propto \rho^{m}$ с показателями $m=2.7-4.3$ и $m=5.5-6$ соответственно. Для классической ГР показатель зависимости $\mu_{s r} \propto \rho^{m}$ составляет $m \approx 5, \mu_{s \_ \text {рh }}(\rho)$ описываются степенной функцией при $\rho<1.7 \mathrm{Hм}$, а при $\rho>1.7 \mathrm{Hм}$ имеют экспоненциальный характер. Компоненты подвижности, связанной с рассеянием на микрорельефе $\Gamma$, в зависимости от центроида заряда изменяются по степенному закону $\mu_{s r} \propto \rho^{m}$ с показателем $m=5.5-6$ и $m=5$. Показано, что структурные несовершенства, вызывающие изменение рассеяния на фононах вблизи классической ГР, существенны в пределах 3.5 нм от гетерограницы.

Получение профилей компонент подвижности важно для физического понимания процессов рассеяния вблизи гетерограниц (определения доминирующего механизма рассеяния) и позволяет анализировать структурное качество пленки в пределах единиц нанометров от тестируемой ГР, что может быть полезно при оптимизации технологических процессов в случае тонкопленочных структур.

\section{Финансирование работы}

Работа выполнена при поддержке проекта Министерства науки и высшего образования (ГЗ № 0306-20190011).

\section{Благодарности}

Авторы выражают благодарность А.К. Гутаковскому за предоставление результатов высокоразрешающей электронной микроскопии, полученных с использованием оборудования ЦКП „Наноструктуры“ ИФП СО РАН (г. Новосибирск).

\section{Конфликт интересов}

Авторы заявляют, что у них нет конфликта интересов.

\section{Список литературы}

[1] J. Hartmann. Proc. 2014 IEEE Compound Semiconductor Integr. Circuit Symp. (CSICS) (La Jolla, USA, 2014) p. 5.

[2] B. Doris, B. DeSalvo, K. Cheng, P. Morin, M. Vinet. SolidState Electron., 117, 37 (2016).

[3] E. Jaberansary, T.M.B. Masaud, M.M. Milosevic, M. Nedeljkovic, G.Z. Mashanovich, H.M.H. Chong. IEEE Photon. J., 5, 6601010 (2013).

[4] Y. Wang, M. Kong, Y. Xu, Z. Zhou. J. Optics, 20, 025801 (2018).

[5] O.V. Naumova, B.I. Fomin, D.A. Nasimov, N.V. Dudchenko, S.F. Devyatova, E.D. Zhanaev, V.P. Popov, A.V. Latyshev, A.L. Aseev, Yu.D. Ivanov, A.I. Archakov. Semocond. Sci. Technol., 25, 055004 (2010).
[6] E. Dmitrienko, O. Naumova, B. Fomin, M. Kupryushkin, A. Volkova, N. Amirkhanov, D. Semenov, I. Pyshnaya, D. Pyshnyi. Nanomedicine, 11, 2073 (2016).

[7] К.Д. Щербачев, В.Т. Бублик, В.Н. Мордкович, Д.М. Пажин. ФТП, 45(6), 738 (2011).

[8] O.V. Naumova, E.V. Vohmina, T.A. Gavrilova, N.V. Dudchenko, D.V. Nikolaev, E.V. Spesivcev, V.P. Popov. Mater. Sci. Eng. B, 135 (3), 238 (2006).

[9] T. Rudenko, A. Nasarov, V. Kilchytska, D. Flandre. Solid-State Electron., 117, 66 (2016).

[10] M. Prunnila, J. Ahopelto, H. Sakaki. Phys. Status Solidi A, 202, 970 (2005).

[11] A.H. Tarakji. Phys. Status Solidi A, 215, 1700419 (2018).

[12] S. Takagi, A. Toriumi, M. Iwase, H. Tango. IEEE Trans. Electron Dev., 41, 2357 (1994).

[13] S. Kaya. Phys. Status Solidi В, 239, 110 (2003).

[14] О.В. Наумова, Э.Г. Зайцева, Б.И. Фомин, М.А. Ильницкий, В.П. Попов. ФТП, 49, 1316 (2015).

[15] G. Hamaide, F. Allibert, F. Andrieu, K. Romanjek, S. Cristoloveanu. Solid-State Electron., 57, 83 (2011).

[16] Э.Г. Зайцева, О.В. Наумова, Б.И. Фомин. ФТП, 51, 446 (2015).

[17] S. Cristoloveanu, N. Rodriguez, F. Gamiz. IEEE Trans. Electron Dev., 57, 1327 (2010).

[18] S. Cristoloveanu, T.V. Chandrasekhar Rao, Q.T. Nguyen, J. Antoszewski, H. Hovel, P. Getntil, L. Faraone. IEEE Trans. Electron Dev., 56, 474 (2009).

[19] C. Lombardi, S. Manzini, A. Saporito, M. Vanzi. IEEE Trans. Comput.-Aided Des. Integr. Circuits Syst., 7, 1164 (1988).

[20] M. Schmidt, M.C. Lemme, H.D.B. Gottlob, F. Driussi, L. Selmi, H. Kurz. Solid-State Electron., 53, 1246 (2009).

[21] J.A. Lopez-Villanueva, P. Cartujo-Cassinello, F. Gamiz, J. Banqueri, A.J. Palma. IEEE Trans. Electron Dev., 47, 141 (2000).

[22] T. Ohashi, T. Tanaka, T. Takahashi, S. Oda, K. Uchida. IEEE J. Electron Dev. Soc., 40, 278 (2016).

[23] K. Uchida, S. Takagi. Appl. Phys. Lett., 82, 2916 (2003).

[24] S. Eminente, S. Cristoloveanu, R. Clerc, A. Ohata, G. Ghibaudo. Solid-State Electron., 51, 239 (2007).

Редактор Л.В. Шаронова

\section{Profiling of mobility components near interfaces of silicon thin films}

\author{
E.G. Zaitseva, O.V. Naumova, B.I. Fomin
}

Rzhanov Institute of Semiconductor Physics, Siberian Branch of Russian Academy of Sciences, 630090 Novosibirsk, Russia

\footnotetext{
Abstract In this paper, we proposed the method for profiling of components of charge carriers effective mobility $\mu_{\text {eff }}$ determined by their scattering by surface phonons and by roughness at film/insulator interfaces. The method is based on the controlled localization of charge carriers relative to the interface under study due to the coupling effect. The proposed method allows us to independently determine mobility components near different interfaces of films. The use of the proposed method for studying the mobility has allowed us to obtain information on interface roughness and on structural quality of ultrathin $(1-3 \mathrm{~nm})$ layer $\mathrm{Si}$ near the Si/buried oxide interface.
} 